\title{
Anthropo-éthologie des non-humains politiques
}

Résumé. La tentation d'interroger les primates en leur posant la question de notre origine reste présente dans le domaine de l'éthologie. Plus largement, on peut remarquer que l'animal se constitue souvent comme un opérateur anthropologique d'identité, soit par similitude, soit par inversion ou contraste. Or, les faits issus des observations traduisent le plus souvent des valeurs, voire reflètent des préférences quant aux modes d'organisation sociale. Ce constat ne doit pas nous conduire au relativisme. Il nous invite au contraire à envisager le savoir de l'éthologie comme un savoir qui construit simultanément l'identité de l'homme et de l'animal, ensemble. Cet article se propose d'explorer les conditions concrètes dans lesquelles ce type de savoir peut se constituer.

Mots-clés. Anthropologie - Organisation humain-animal - Origine - Primates Valeurs

Abstract. The temptation to seek in primates our own origin is still found in ethology. More broadly speaking, we see that the animal kingdom is often used as an antbropological operator of identity, using either similitude or inversion or contrast. The observation data most often reflect values, or even preferences, concerning modes of social organization. However, this observation should not lead to relativism. On the contrary, it invites us to envisage ethological knowledge as constructing humans and animals at the same time, together. This article sets out to explore the concrete conditions in which this kind of knowledge can be constructed.

Key words. Anthropology - Human-animal organization - Origin - Primates Values

Information sur les Sciences Sociales (C) 2006 SAGE Publications (Londres, Thousand Oaks, CA et New Delhi), 0539-0184

DOI: $10.1177 / 0539018406063635$ Vol 45(2), pp. 209-226; 063635 
"L'ethnologie pourrait permettre de reprendre en termes nouveaux la réflexion sur l'histoire, pourvu qu'on y cherche non un accès à des formes primitives d'une évolution humaine, mais plutôt les éléments d'une confrontation entre des types de devenir", écrivait, il y a presque 30 ans d'ici, le philosophe politique Claude Lefort (1978: 32). S'il semble bien que ce programme, qui rompait avec certaines tendances en ethnologie, ait ouvert une voie prometteuse à la constitution d'une anthropologie politique qui modifie radicalement son approche des autres cultures - et de la nôtre - on pourrait se demander si l'éthologie ne devrait pas, à son tour, envisager d'opérer ce même renversement des questions.

Certes, certains des éthologues et plus particulièrement des primatologues ont déjà redéfini leur programme de recherches dans les termes d'une "confrontation des types de devenirs"; n'est-ce pas ce que tente Shirley Strum à l'issue de son travail avec les babouins lorsqu'elle écrit:

L'observation de la bande de Pumphouse conduit à la même conclusion: rien ne prouve que l'agression, la supériorité des mâles et leur mainmise sur le pouvoir politique soient caractéristiques du mode de vie des premiers humains. D'autre part, si l'on croit sincèrement que la société des humains est caractérisée par la loi du plus fort, la supériorité masculine et une hiérarchie stable entre les mâles, alors il faudra bien trouver de nouvelles explications. Nous ne pouvons plus nous contenter de dire qu' "il en va ainsi" de toute société. (1990: 114)

Il ne s'agit pas de prétendre que les babouins n'ont rien à voir avec les humains et de rappeler la coupure, bien au contraire; il s'agit de témoigner de l'inventivité des figures du social, dont aucune ne peut être reléguée au statut de formes primitives ou inachevées de nos modes d'organisation; il s'agit de faire que leur histoire ne soit pas simple précurseur de la nôtre.

Cette tentative est cependant loin de faire l'unanimité et l'éthologie semble, à cet égard, bien loin des nouvelles orientations de l'anthropologie: il n'est pas rare en effet, encore aujourd'hui, de voir convoquer l'animal pour lui demander de suppléer à la défection de ceux à qui revenait le rôle de témoigner de notre origine: ceux qu'on a d'ailleurs longtemps appelés les primitifs. Ainsi, les questions adressées à l'animal (comme elles l'étaient au "primitif" à une certaine époque) semblent souvent subordonnées à l'espoir d'élucider le mystère de l'origine, que ce soit celle de la moralité, de l'organisation sociale, de la répartition des rôles et du pouvoir, voire du contrat social. 
Les critiques de plus en plus nombreuses n'ont cependant pas manqué de souligner la singulière inversion qui sous-tendait ces histoires et ces fictions: les animaux ne témoignaient si bien d'une histoire de l'origine que parce qu'on donnait, a priori, aux observations les significations mêmes de l'histoire qu'il s'agissait de retrouver. On se souviendra de cette joyeuse boutade du philosophe Bertrand Russell (1961: 160), s'étonnant de ce que les animaux "apparemment, se conduisent toujours de manière à prouver la justesse de la philosophie de l'homme qui les observe". En témoigne, dit-il, le fait qu'au XVIIIème siècle "les animaux étaient féroces, mais sous l'influence de Rousseau, ils commencèrent à illustrer le culte du Noble Sauvage dont Peacock se moque dans OurangHaut-ton. Pendant tout le règne de la Reine Victoria, les singes furent de vertueux monogames, mais durant les années 20, leurs mœurs se détériorèrent d'une manière désastreuse" (1961: 161).

Les critiques féministes, quant à elles, ont montré, de manière exemplaire, à quel point les schèmes qui guident les observations sont imprégnés des conceptions de l'organisation des rôles et du pouvoir véhiculées par la culture de l'observateur; que l'on pense par exemple à la différence de ce qui était observé chez les primates par Zuckerman au zoo de Londres dans les années 1930 et ce que des chercheuses comme Linda Fedigan, Thelma Rowell, Barbara Smuts et Shirley Strum nous rapportent aujourd'hui: ${ }^{1}$ autres temps, autres observations, autres contraintes sur les significations. Avec les premières recherches, on en revient toujours, comme l'écrit Strum (1990: 106), "à une société dominée par les mâles, caractérisée par une division claire du travail; une société où les mâles détiennent le pouvoir et où les femelles ne peuvent être promues qu'en s'associant à un mâle dominant". On constate en outre que ces observations s'inscrivent, dès les premiers travaux de Darwin et pendant les décades qui suivirent, dans un réseau de référents plus littéral qu'analogique, les deux figures de l'ancêtre: le sauvage et l'animal. Qu'on relise les travaux du Darwin de La descendance, ${ }^{2}$ ceux de Freud (1912) inventant sa fiction dans Totem et tabou et de bien d'autres encore: politique des genres, politique des rapports de pouvoir et politique colonialiste convergent dans un même motif, histoire naturelle et anthropologie traduisent une complicité qui trouvait, à l'époque, de multiples justifications.

Certes, à cette ancienne complicité entre l'anthropologie et l'histoire naturelle s'est substituée aujourd'hui une autre manière d'articuler la question animale et la question anthropologique. Dans 
cette articulation, nouvelle dans certaines de ses formes, ancienne dans d'autres, l'animal constitue ce que nous pourrions nommer un "opérateur anthropologique d'identité": c'est à lui que sera demandé de constituer l'identité de l'homme, qu'il traduise par la construction de contrastes, la singularité de celle-ci, ou, au contraire, qu'il témoigne, par un jeu de similitudes, de l'enracinement de cette identité dans la nature. La première opération se décline dans les figures de l'inversion, la seconde dans les procédures de "naturalisation".

\title{
Figures de l'inversion
}

"Il y a", écrit le naturaliste américain Bruce Bagemihl en reprenant une phrase de Weinlich,

\begin{abstract}
une longue et sordide histoire des affirmations de l'unicité de l'homme. Sur toutes ces années, j'ai lu que les humains étaient les seules créatures qui rient, qui tuent d'autres membres de leur propre espèce, qui tuent sans le besoin de manger, qui présentent une réceptivité sexuelle des femelles permanente, qui mentent, qui montrent un orgasme féminin, qui tuent leur propre jeune. (1999: 46)
\end{abstract}

Il ne sera pas inutile d'ajouter le commentaire ironique de Bagemihl concluant que, peut-être, "la seule vraie différence entre les espèces est que les gens, au contraire des animaux, ont tendance à faire des généralisations simplistes".

On doit le reconnaître, ces généralisations simplistes ont foisonné dans une certaine littérature. Le discours qui prône la rupture a fait bien des émules. Quantité de justifications sont convoquées pour étayer la nécessité du maintien de la frontière humain-non-humain - ou nature-culture. On pensera plus particulièrement à celles avancées par une tradition humaniste invoquant les leçons de l'histoire la rupture garantit le respect de l'humain; ou encore aux arguments de certains courants de pensée influencés par l'anthropologie affirmant qu'il s'agirait d'une tendance "naturelle" présente dans toutes les cultures, en misant sur l'univocité - et donc l'universalité - de la définition de la nature; voire encore à l'argument apparemment sans appel: les hommes ne sont pas des animaux.

Cependant, derrière l'apparente diversité de ces arguments prônant ou justifiant la volonté de rupture, un même enjeu se dessine: celui de produire, en creux, et par le détour du négatif, une définition positive: l'homme, au contraire de l'animal . . L'identité de l'un se définit par les manques et les inachèvements de l'autre. ${ }^{3}$ 
Les recherches féministes de ces dernières années, comme celles qui leur ont emboîté le pas en analysant la construction de la figure de l'homosexualité, avaient déjà montré le véritable motif à l'œuvre dans ce type de stratégie: la femme comme l'homosexuel offre une figure de l'inversion qui autorise à produire, par contraste, en négatif, une définition positive d'un universel: l'homme, dans le premier cas, l'hétérosexuel dans le second.

Envisager l'animal comme une figure de l'inversion, sur le mode de ce que nous ne sommes pas, ou plus, ce à quoi nous avons réussi à nous arracher, produit de fait un résultat très similaire. $\mathrm{La}$ question qui est en jeu apparaît bien la même: "comment définir ce qui doit se constituer comme une norme (dans ce cas, l'humain) sans laisser transparaître la contingence de celle-ci?". En outre, dans la multiplicité des versions possibles, si vous essayez de produire une définition positive de l'identité humaine, vous risquez toujours de voir brutalement surgir un trouble-fête, une culture minoritaire, un collectif féministe, un groupe d'homosexuels, de noirs ou de natifs Américains qui viendront crier "Pas nous, pas nous!". "Nous ne pouvons adhérer à ce "nous' de la proposition de définition de l'être humain", "votre définition vient de nous exclure du collectif!" Dès lors, devant cette impossibilité de stabiliser une définition, l'animal va être appelé à la rescousse: il va venir donner le négatif de la définition, négatif qui, de la manière même dont il est défini, pourra ambitionner de mettre tout le monde plus ou moins d'accord: "voilà ce que nous ne sommes pas (ou plus)"; "voilà ce que nous sommes les seuls à pouvoir". L'animal va être enrôlé comme un collectif, qui, par son exclusion, permet à l'être humain de se créer une identité par contraste. Il s'agit en somme de construire un réservoir d'universalité: en témoigne la sémantique particulière utilisée dans ce cadre. On ne parle pas des animaux, des babouins, des rats ou des drosophiles, mais de l'Animal. Rencontre de deux universels singuliers: l'homme au contraire de l'animal. . . .

Cette tradition de hiérarchisation misant sur la rupture a bien sûr rencontré, au cours de ces dernières années, des démentis de plus en plus troublants. ${ }^{4}$ Nous ne sommes pas les seuls à rire, nous apprend de Waal, après avoir provoqué l'hilarité de ses chimpanzés en se déguisant en léopard. ${ }^{5}$ L'infanticide, l'orgasme des femelles, le mensonge, la conscience de soi, la réconciliation, la capacité d'attribuer des intentions aux autres, l'outil, le langage, l'accès aux symboles, la liste est longue, semblent devoir de mieux en mieux se partager. Nous sommes de moins en moins seuls au monde. 


\section{Naturalisation}

Le problème de cette reconnaissance progressive des similitudes, cependant, ne peut se donner comme une solution; paradoxalement, elle ne prémunit pas mieux l'animal de ce rôle d'opérateur anthropologique. Les choses se compliquent d'autant plus que cette proximité de plus en plus grande favorise souvent la tentation de retrouver, dans la nature, non seulement l'élucidation d'une origine, mais la légitimation d'une certaine normativité. L'animal y devient porteur non seulement d'une histoire (qui est la nôtre) mais aussi de valeurs, qui parce qu'elles émergent de la nature, acquièrent une force et une légitimité inégalées. ${ }^{6}$ Certes, la plupart des auteurs reconnaissent la distinction faits-valeurs en affirmant qu'on ne peut établir de valeurs à partir de faits, du devoir-être à partir de l'être; mais cette distinction philosophiquement honorable, on s'en rend compte à l'analyse, n'est là que pour masquer que le problème de la confusion faitsvaleurs ne se situe pas seulement à ce niveau, et que la tentation de créer des normes au départ de la nature n'en est que la version la plus naïve - et la plus repérable.

Un exemple issu d'un extrait de Frans de Waal illustre bien comment, en se prémunissant contre le premier piège, celui qui consiste à établir une normativité à partir de faits, on tombe, justement à cause de cette séparation, immanquablement dans un second, celui qui consiste à abriter en douce des valeurs derrière des observations apparemment objectives. Lorsque de Waal (1995: 208-9) décrit la manière dont se répartissent les rôles et les statuts dans la société de chimpanzés qu'il observe, il note que les mâles présentent des activités stratégiques orientées vers le statut, en accord, dit-il, avec "une politique dont l'objectif est l'accroissement de pouvoir". Les femelles, par contre (comme les enfants), "présentent des interactions qui s'accordent avec leur sympathie". Les femelles sont intelligentes, certes, mais elles ne présentent pas la même qualité de rationalité que les mâles: à ces derniers l'espace public et politique des stratégies de pouvoir, fondées sur la rationalité, aux premières l'espace de la socialisation, fondée sur les sentiments. De Waal se demande néanmoins, sans ingénuité, si ses conclusions ne sont pas la marque de ses préjugés. Bel effort de réflexivité. Mais cette louable tentative s'étouffe dans l'œuf et révèle tout à coup les véritables enjeux de tout ceci. Je cite de Waal, car on ne peut s'exprimer plus clairement: 
On prétend qu'il est réactionnaire de considérer les différences qui existent entre les sexes dans le comportement humain comme héréditaires, sous prétexte que, si cela était vrai, nous serions condamnés à obéir éternellement à notre nature. Cette conclusion pessimiste est cependant fausse. L'homme a prouvé que le respect des lois naturelles n'empêche pas de les dépasser. Les avions, par exemple, paraissent se moquer de la gravité, mais ils ont été conçus par des gens qui reconnaissent cette force et en ont étudié les effets. (1995: 209)

Le coup de l'avion est admirable. On va obéir à la nature pour mieux la faire obéir! De Waal reprend la plus vieille stratégie des scientistes: on vous donne les faits, on vous laisse libres quant aux valeurs. Qu'allons-nous demander à ce que nous appelons "nature"? Des lois auxquelles nous référer, même si c'est pour prendre certaines libertés avec elles. Comment comprendre autrement ce que vient faire cette "nature" ainsi convoquée dans cette affaire? Il faut être attentif à la manière dont de Waal la mobilise: il commence par établir une claire distinction entre questions "sociales" (son effort de réflexivité ne traduit pas autre chose) et questions "naturelles". Cette distinction ainsi organisée n'est cependant pas là pour empêcher que l'on passe de l'une à l'autre: au contraire elle n'est là que pour permettre ce passage, mais subrepticement. Il y a un tri dans ce passage: d'un côté la nature et ses objets, les faits; de l'autre les subjectivités et les préjugés, les valeurs. ${ }^{7}$ En d'autres termes, les faits, parce qu'ils sont bien séparés des questions de valeur - les faits ne vous imposent rien, si ce n'est de leur obéir pour les transgresser - acquièrent le pouvoir de s'imposer en toute innocence.

Or, s'il y a bien quelque chose que l'histoire de l'éthologie nous a appris, et que soulignait tant la boutade de Russell décrivant la dégradation désastreuse des moeurs d'animaux autrefois bien victoriens que les critiques féministes, c'est qu'il n'y a pas de faits sans significations, sans préférences, et que ces dernières précèdent les faits. Attribuer à des animaux, qui implicitement renvoient à nous, sur la base de leur appartenance sexuelle, tantôt des conduites caractérisant le registre social (pour les femelles), tantôt des conduites relevant du registre politique (pour les mâles), reconnaître aux unes des motifs émotionnels et aux autres des registres plus stratégiques ou rationnels, dans une culture qui opère, sur la base de la rationalité, la séparation de l'espace public, donc de l'espace du pouvoir, et de l'espace privé, n'a rien d'un fait, et n'a surtout rien de neutre ou d'innocent: il s'agit déjà et toujours de significations qui mettent en jeu des valeurs, qui pérennisent des normes. 
La difficulté des recherches s'ingéniant à retrouver, dans une nature "des origines", une origine à notre identité, tient finalement à cette situation paradoxale: tout dépend des valeurs à l'origine de ce que vous cherchez. Une boutade circule chez les éthologistes selon laquelle Marx aurait eu parfaitement raison quant à la possibilité du socialisme, ... il se serait simplement trompé d'espèce. Vous pouvez reconstruire une histoire morale de la nature, il suffit de bien choisir les héros de votre histoire, les moments d'observation, les comportements et les interprétations: on n'oubliera pas l'étonnant contraste entre Kropotkine, naturaliste anarchiste russe, et Darwin, le premier voyant dans la nature les preuves des vertus de la solidarité du point de vue de la survie et des lois de la sélection naturelle, là où le second insistait sur l'importance de la compétition. ${ }^{8}$ Il ne s'agit pas d'affirmer simplement que ces deux auteurs n'ont vu dans la nature que ce que leur position historique particulière ${ }^{9}$ les conduisait à observer; le fait que le premier menait ses recherches dans de vastes espaces qui permettent aux animaux d'éviter la surpopulation alors que le second avait été confronté à des populations insulaires (où le problème se pose de manière aiguë) n'est pas sans importance (Todes, 1988). On pourra encore citer en exemple le "tournant moral" pris par l'éthologie après les recherches de Lorenz: la mort avait quasiment disparu de la nature. ${ }^{10}$ L'intérêt de Lorenz pour les mécanismes qui président à la formation des liens et pour les stratégies qui régulent la violence a largement favorisé certaines observations - et certaines espèces. Ainsi, les affirmations de Lorenz (1969) selon lesquelles l'agression débouche rarement sur la mort se fondaient sur les observations de certains comportements - surtout les rituels - dont la fonction était, selon lui, d'inhiber et de réorienter l'agression intra-spécifique.

Un monde moral peut être ainsi reconstruit, il suffit d'en chercher l'histoire et les témoignages. Mais vous pourriez tout aussi bien déboucher sur une tout autre façon de faire l'histoire, et soit vous retrouver à célébrer les vertus de l'esclavage, à légitimer l'homophobie, l'agression ou la violence soit, de manière plus sophistiquée, arriver, comme le fait de Waal, à naturaliser la domination des mâles sur les femelles, en invoquant une plus grande rationalité chez les premiers. ${ }^{11}$ Bref, pour plagier la belle ironie de Darwin, vous pourriez tout aussi bien vous retrouver avec une nature écrite par le chapelain du diable. ${ }^{12}$ 


\section{Identités partagées}

Est-ce dire alors, au vu de tout ce qui précède, que nous devions renoncer, une fois pour toutes, à demander à l'animal de participer à la définition de l'humain? Cette résolution pourrait, en apparence, paraître sage. Mais elle nous ferait manquer alors une des plus intéressantes ressources de notre histoire avec les animaux: le fait que nous nous sommes fabriqués avec eux. Entre différence radicale figure de l'inversion - et modèle anthropologique, il existe une autre voie, que les travaux de Dominique Lestel (1996) nous invitent à explorer. Ce que les communautés hybrides proposent de repenser de fond en comble, c'est la notion même d'identité. Il ne s'agit plus de la traduire, soit de manière abstraite, en référence à elle-même, soit, de manière tout aussi abstraite, par contraste ou par analogie, mais de penser les identités comme des formes du devenir ensemble. Non plus chercher des référents universels, mais explorer avec appétit des situations locales et concrètes: il ne s'agit dès lors plus de définir des identités - définir, c'est l'étymologie qui nous l'apprend, revient à mettre des limites - mais d'explorer des élargissements de répertoires. En d'autres termes, l'éthologie et l'anthropologie ont quantité de choses et d'expériences à construire ensemble dans l'exploration des formes de devenir avec l'humain pour l'animal, avec l'animal pour l'humain.

Il s'agit en somme de concevoir une anthropologie politique qui puisse prendre en compte des modes d'organisation des humains et des animaux, mais en renonçant à le faire du point de vue des seuls humains. ${ }^{13}$ Comment les modes d'organisation modifient-ils les répertoires de chacun, hommes et bêtes qui sont amenés à cohabiter, à s'intéresser l'un à l'autre; comment s'affectent-ils mutuellement; qu'est ce qui change du fait de privilégier tel ou tel type de vivre ensemble? Les situations à explorer sont multiples, marquées d'inventivité et de contingence. Comment les corbeaux mènent-ils les Esquimaux à s'organiser avec eux, et qu'est ce que cela change pour les corbeaux (Heinrich, 1991)? Comment des expériences scientifiques avec des rats vont-elles conduire les chercheurs à totalement reconsidérer la pertinence des questions qu'on leur adresse usuellement, sous l'influence d'une compétence surprenante: les rats reconnaissent leurs scientifiques, manifestent des préférences et modifient leurs conduites en fonction de celui qui adresse ses questions. ${ }^{14}$ Certains des chercheurs en éthologie ou en psychologie animale ont depuis peu reformulé la singularité de leur travail: 
"ce que nous étudions", disent-ils, "ce sont des liens. Ce sont en dernier ressort, les relations entre un observateur et un observé qui constituent les données scientifiques" (Fentress, 1992: 45).

Une situation me paraît aujourd'hui exemplaire de l'intérêt de cette proposition en termes de recherches des "formes de devenir ensemble", dans la mesure où elle se constitue d'une part comme une véritable expérimentation sur l'élargissement des répertoires, ce qui définit le but de la recherche, et, d'autre part, sur la quête des bonnes manières de s'organiser entre humains et animaux.

Thelma Rowell est une primatologue anglaise qui a décidé de consacrer ces dernières années à l'étude du mouton. Après une première étude avec un groupe élevé dans le parc de l'université de Berkeley, où elle enseignait, elle les observe à présent dans la prairie en face de chez elle, dans le nord du Yorkshire.

Les observations de la primatologue commencent en général tôt le matin. Elles débutent par le même rituel: la chercheuse apporte à ses 22 moutons les bols du petit déjeuner. Cela lui permet de les observer de près, cela tend aussi à rendre les comportements un peu plus nombreux (les moutons sont des animaux qui, par nature, tendent à rester discrets) et un peu plus visibles: il est plus facile de les approcher pendant ce moment. Ce qui est intéressant c'est que Thelma Rowell apporte 23 bols à ses 22 moutons. Il y en a toujours un de trop.

Pourquoi ce bol surnuméraire? Avant de répondre à cette question, il nous faut préciser les enjeux de la recherche. Au départ, Thelma Rowell, qui avait consacré presque toute sa carrière aux singes, s'est inquiétée de ce qu'elle appelle un "scandale hiérarchique": les singes deviennent, au fur et à mesure des recherches, de plus en plus intelligents, si on les compare à quantité d'autres animaux. Le mouton est, à cet égard, un cas presque paradigmatique puisqu'on ne lui reconnaît que très peu de compétence sociale, et encore moins d'intelligence. Le scandale hiérarchique ne serait-il pas simplement l'effet d'un artefact des recherches?

En effet, on ne peut manquer que les chercheurs ont adressé des questions sociales, intelligentes, sophistiquées aux singes; aux autres, ils ont demandé comment ils se nourrissent, de quoi ils ont peur, voire comment ils organisent la hiérarchie; en somme, des questions qui ne leur ont pas laissé beaucoup de chances de montrer leurs compétences.

La primatologie, en d'autres termes, a progressivement adopté, au cours de l'évolution des recherches, les méthodes de l'anthropologie 
et en a emprunté les questions. L'éthologie classique, par contraste, s'est largement focalisée sur les relations avec et autour de la nourriture: qui mange quoi, comment les animaux s'organisent-ils autour des ressources, etc.? Ces différences de méthodes renvoient elles-mêmes à des enjeux divers, liés, notamment, aux animaux eux-mêmes, aux différentes questions qu'ils suscitent, à des problèmes de pratique et de terrains, etc. Les primates, considérés comme nos proches parents, voire comme les ancêtres de l'évolution humaine, ont mobilisé leurs chercheurs autour de questions sociales. Certes, eux-mêmes encourageaient leurs chercheurs, à cet égard, et cela est d'autant plus lisible si on observe ce simple contraste: la plupart des animaux passent une bonne partie de leur temps à ne rien faire; chez les primates, au contraire, il se passe toujours quelque chose, ils sont toujours occupés à se chamailler, à s'épouiller, à entrer en relation. Ils sont donc non seulement plus amusants à observer, mais la récolte de données nécessaires à une recherche qui ne soit pas considérée comme une simple collection d'anecdotes, va dès lors considérablement varier. La différence essentielle ne tient donc pas tellement à une différence de sophistication, mais plutôt à une différence de rythme. Celle-ci, dans le cadre des projets de recherche, va peser lourdement.

L'intérêt de l'éthologie classique pour les comportements alimentaires, par opposition à la primatologie, plus orientée vers les questions sociales, peut également s'expliquer dans les termes de cet "artefact" de l'intérêt des chercheurs. ${ }^{15}$ En effet, selon Thelma Rowell, le privilège accordé par l'éthologie classique aux problèmes liés aux ressources alimentaires tient principalement aux conditions mêmes de l'observation: les comportements alimentaires sont beaucoup plus faciles à observer. Ceci a conduit les chercheurs à s'intéresser aux relations autour de la nourriture, et plus particulièrement aux relations de compétition autour de cette dernière. ${ }^{16}$ Cette habitude a conduit les éthologistes à largement surestimer l'importance de la compétition dans la nature. Or, ce qui est important pour les animaux, affirme-t-elle, ce qui les mobilise le plus, n'est pas tellement de manger mais d'échapper au fait de l'être soi-même. Ce qui est important est cependant beaucoup plus rare: c'est la prédation. Mais la rareté des observations de prédation n'est ellemême pas étrangère à un artéfact: on ne voit que rarement les animaux que nous observons lutter contre un prédateur. Et ce, pour une bonne raison: du fait de notre présence! Quand nous sommes avec eux, nous constituons une sorte de protection, et nous tenons 
les prédateurs à distance, et les animaux que nous observons s'en rendent compte, et l'exploitent. C'est finalement, conclut la chercheuse, ce que nous appelons "habituation". Ainsi, les petits singes bleus qu'elle a observés en Afrique sont la proie des aigles; on les voit planer continuellement au-dessus des arbres où vivent les singes. Qu'est ce que voit un aigle lorsqu'il jette un regard vers le bas? Des visages humains derrière des paires de jumelles tournées vers le ciel. Cela suffit à les dissuader et à les convaincre d'aller chercher leurs proies dans un autre groupe.

Or, et c'est là le problème, entre la compétition et la défense contre le prédateur réside une différence essentielle: les animaux s'organisent tout autrement dans chacune de ces situations. Les comportements mobilisés demandent peu de compétence sociale dans le premier cas, en revanche, ils témoignent, dans le second, d'un tout autre niveau d'organisation puisque les animaux sociaux vont, très souvent, se mobiliser collectivement contre le prédateur (alarmer les autres, protéger les petits, voire organiser une défense commune). En d'autres termes, selon que vous vous intéressez à l'un ou l'autre aspect de la vie des animaux (se nourrir, se défendre) vous aurez des animaux très différents, et des versions de l'organisation sociale peu sophistiquées dans un cas, beaucoup plus dans l'autre.

Pour en revenir à nos moutons, ce contraste conduit à envisager que, selon l'un ou l'autre des dispositifs, selon le choix des questions, les moutons recevront - ou ne recevront pas - la chance de témoigner de compétences sociales. Il s'agit dès lors d'apprendre à repérer ce qu'il faut observer pour répondre à la question de ces compétences. La première de ces questions serait de savoir s'ils sont capables, comme les primates ont pu le montrer, de nouer des relations de longue durée. La possibilité, pour les moutons, de répondre à cette question, dépend elle-même de la qualité du dispositif offert.

Car certaines recherches s'y étaient déjà attelées: et elles ont répondu par la négative. Mais à l'analyse, on s'aperçoit tout de suite que les conditions mêmes rendaient peu probable la possibilité, pour les moutons, de manifester des comportements sociaux sophistiqués. D'abord, la plupart des recherches ont été menées avec des groupes constitués pour l'expérience, les animaux achetés pour la circonstance ne s'étaient jamais rencontrés auparavant: il aurait fallu un miracle pour voir apparaître des liens durables.

Ensuite, une bonne part des recherches ont monopolisé leur question en prenant pour critère d'organisation sociale celui de la 
hiérarchie. Ce qui aboutit, comme dans le travail de l'éthologiste Geist (1971; cité par Rowell et Rowell, 1993: 219), à une description relativement simple des comportements, dont la hiérarchie constitue en définitive le seul principe organisationnel. Le mâle dominant conduit le troupeau, suivi par les autres mâles et puis les femelles. Les relations entre les individus sont déterminées par la taille des cornes, elle même tributaire de l'âge et du sexe. La reconnaissance individuelle n'est pas nécessaire dans ce système - ce qui est, note Thelma Rowell, une réminiscence des premières descriptions des organisations de primates. Les comportements se limitent généralement à des conflits entre mâles. En somme, ces moutons font ce qu'on peut attendre d'une part, des moutons - se suivre les uns les autres de manière très prévisible - et ce qu'on peut attendre, d'autre part, des animaux répondant aux théories de la hiérarchie: gagner le droit de conduire à grands coups de cornes, les mâles devant, les femelles derrière.

Or, souligne Thelma Rowell, ces moutons certes, se comportent de cette manière ... un mois par an, au moment de la reproduction, et c'est le moment qu'a privilégié Geist parce que c'est le moment où les moutons sont les plus actifs. Ce qu'il décrit comme constituant le comportement habituel des moutons s'avère cependant, si on les observe au cours des 11 mois restant, totalement différent: d'une part, c'est la femelle la plus âgée qui conduit le troupeau, d'autre part, mâles et femelles ont des systèmes sociaux peu semblables et relativement indépendants l'un de l'autre.

La théorie de la hiérarchie, issue de l'éthologie classique et qui a constitué le fond paradigmatique de nombreuses recherches (Haraway, 1992), semble elle-même une condition qui, quoiqu'elle offre une certaine visibilité à certains phénomènes, comme celui du leadership, ne permet pas de rendre compte de comportements sociaux sophistiqués. Un seul principe organisateur, c'est à la fois trop et trop peu - cela peut rendre compte de tout, et donc barrer la route à tout autre hypothèse. Ce modèle octroie peu de chances aux moutons: les voilà plus moutonniers que jamais, non seulement ils sont immuablement contraints de suivre les autres, mais ils sont en outre tout aussi immuablement contraints de suivre des règles rigides, d'autant plus inflexibles qu'elles dépendent de la taille des cornes. La vision d'un groupe d'individus déterminés par une organisation strictement hiérarchisée laisse finalement peu de place à la flexibilité et à la sophistication. Deux moutons qui s'affrontent à grands coups de cornes, c'est une question de hiérarchie; un 
mouton qui guide, c'est le signe de sa place dans la hiérarchie. Certes, on a pu constater chez les femelles une organisation semblable à celle qu'on appelle hiérarchie; c'est toujours la plus âgée qui donne le signal de départ, les autres la suivront. Cependant, la notion de hiérarchie, telle qu'elle est généralement comprise dans sa fonction de tenir le groupe ensemble, occulte la manière dont cette organisation s'effectue chez les brebis (comme d'ailleurs chez les chimpanzés pour lesquels Margaret Power [1991] suggère de remplacer le terme "dominant" par celui de "leader charismatique"): il n'y a aucune coercition.

La manière dont les mâles s'organisent, en revanche, s'avère beaucoup plus imprévisible: avant chaque mouvement de déplacement, un des mâles de la troupe a fait un geste, quasiment imperceptible pour nous, qui consiste à dresser légèrement la tête et à pointer son museau dans une direction. Parfois, le groupe se met en marche, parfois non; jusqu'à ce qu'un autre mâle reproduise un geste semblable et, éventuellement, entraîne le groupe dans la direction indiquée.

Une fois qu'on laisse de côté l'explication en termes de hiérarchie, pour les mâles, ou qu'on la restreint à quelques comportements, beaucoup de choses commencent à recevoir non seulement une nouvelle visibilité, mais une lisibilité inédite. Sans la hiérarchie, les animaux, comme les chercheurs, sont beaucoup plus libres, plus inventifs et plus sophistiqués. Sans la hiérarchie, en d'autres termes, les animaux ne sont plus contraints à la répétition - et leurs scientifiques de, ce fait, y échappent également: ils peuvent être mobilisés par d'autres problèmes, comme ceux de la préservation des liens, même en période de rivalité, ceux des affinités, ceux de la réconciliation dont les moutons semblent experts.

Thelma Rowell cherche les conditions qui traduisent les compétences de ces moutons. Nous en revenons à présent à notre question, celle de la raison de la présence de ce fameux bol surnuméraire: il prend, dans cette perspective, tout son sens. Il doit certes permettre d'observer certaines choses, mais il doit surtout éviter de bouleverser les relations: c'est un artefact destiné à empêcher la présence d'un autre, celui qui serait créé par l'apport d'une ressource trop peu importante qui provoquerait immanquablement la compétition. Il ne s'agit pas d'empêcher les moutons d'entrer dans ce type de relation, il s'agit de leur en laisser le choix. Et ceci, non pas au nom d'un motif politique tributaire d'un contexte social particulier (nous préférerions des moutons coopératifs à des moutons compétitifs 
parce que moralement, c'est plus acceptable) mais au nom d'une pratique fondée sur l'intérêt, cette fois au double sens du terme: au sens de prendre soin, faire attention - ne pas créer de conditions sources de stress pour les moutons; au sens également de créer des événements vecteurs d'intérêt, des événements qui permettent de susciter des conduites intéressantes.

La question de l'organisation des moutons se subordonne à celle d'une organisation humains-moutons. C'est une question qui transforme les identités: ce bol surnuméraire doit permettre d'élargir le répertoire d'hypothèses et de questions qui sont proposées aux moutons. Car s'ils choisissent, malgré tout, la compétition, il faudra envisager d'autres explications, plus compliquées que celle de la rareté des ressources: il faudra leur poser d'autres questions sur leur comportement social. Ainsi si vous voyez un mouton quitter son bol, bousculer son voisin pour prendre sa place et revenir tout de suite à la sienne, ou encore persévérer et suivre l'autre pour le déloger à nouveau, vous pourrez faire quantité d'hypothèses, sauf la moins intéressante et la plus prévisible. L'élargissement du répertoire des motifs possibles engage à présent à des explications beaucoup plus sophistiquées. Ce mouton a-t-il voulu simplement montrer à son congénère - et aux autres - qu'il pouvait supplanter? Si tel est le cas, nous avons une hypothèse qui nous montre que les moutons, comme les primates, les corbeaux de Bernd Heinrich (1991, 2000) et les cratéropes de Zahavi, ${ }^{17}$ ont une conception très compliquée de la hiérarchie, et que celle-ci n'a plus rien d'un ordonnancement rigide qui détermine de manière prévisible les conduites.

Créer les meilleures conditions, donner une chance à l'imprévisible, construire un éthos qui favorise les répertoires les plus variés, chercher les significations les plus intéressantes qui ouvrent d'autres hypothèses et d'autres questions, se mettre en appétit par rapport à la diversité, inventorier de multiples formes de devenir, poser la question de la responsabilité de celui qui observe par rapport à ceux qu'il interroge; en somme, chercher les manières de s'organiser qui donnent une chance aux compétences, tant de la chercheuse que de ses animaux. D'une certaine manière, n'est-ce pas ce qu'aujourd'hui, l'anthropologie, comme science vraiment humaine, peut traduire comme réussite?

Vinciane Despret enseigne au Département de Philosophie de l'Université de Liège. Son thème de recherche actuel est la philosophie des pratiques de la psychologie humaine et animale et de l'éthologie. Elle a notamment publié: Quand le loup 
habitera avec l'agneau (Paris: Les Empêcheurs de Penser en Rond, 2001); Hans, le cheval qui savait compter (Paris: Les Empêcheurs de Penser en Rond, 2004). Adresse de l'auteur: Université de Liège, Département de philosophie, Place du XX Août, 9, B. 4000 Liège, Belgique. [email: v.despret@ulg.ac.be]

\section{Notes}

1. Voir à ce sujet Strum et Fedigan (2000: 358-82) et Haraway (1992).

2. "J'aimerais autant pour ma part", écrit Darwin dans ses conclusions, "descendre du petit singe héroïque qui brava un terrible ennemi pour sauver son gardien, ou de ce vieux babouin qui emporta triomphalement son jeune camarade après l'avoir arraché à une meute de chiens étonnés, - que d'un sauvage qui se plaît à torturer ses ennemis, offre des sacrifices sanglants, pratique l'infanticide sans remords, traite ses femmes comme des esclaves, ignore toute décence, et reste le jouet des superstitions les plus grossières" (1981: 678).

3. Ce qui n'est évidemment pas sans rappeler le geste de définir la modernité ou la civilisation par l'inachèvement ou les manques des peuples dits "non-civilisés".

4. Voir pour une analyse de ces multiples transformations Lestel (2001).

5. Anecdote racontée lors d'une soirée thématique diffusée sur la chaîne de télévision ARTE et relayée par D. Lestel (2001: 205).

6. Voir à ce sujet la belle analyse du déplacement de la légitimation de la domination, du domaine religieux au domaine de la nature, dans l'article de Eleni Varikas (2000).

7. On trouvera une version beaucoup plus sophistiquée de cette analyse par Bruno Latour $(1999,2001)$.

8. Ce contraste, comme tous les contrastes rapides, ne rend pas justice à Darwin qui, à de nombreuses reprises, a insisté sur la possibilité de stratégies alternatives à la compétition.

9. Ce fut d'ailleurs un des reproches qu'adressa Marx à Darwin quand il écrit, en 1862, qu'il est remarquable "de voir comment Darwin reconnaît chez les animaux et les plantes sa propre société anglaise, avec sa division du travail, sa concurrence, ses ouvertures et ses nouveaux marchés, ses 'inventions' et sa malthusienne 'lutte pour la vie", (1973: 21).

10. "Il y a", expliquent Avishag et Amotz Zahavi (1997: 15), les spécialistes israéliens des oiseaux cratéropes, "une opinion assez communément partagée, à laquelle souscrivait Konrad Lorenz, et selon laquelle la tendance à monter dans l'escalade des conflits dans les combats et la facilité de tuer son adversaire est unique chez les humains".

11. L'importance que notre tradition accorde à la rationalité, véritable compétence de l'espace public, et la disqualification dont les processus émotionnels font l'objet, montre que la répartition des caractéristiques "psychologiques" correspond en fait à des jeux de pouvoir. Les hommes blancs civilisés se verront attribuer la raison, les femmes, les enfants et ceux qu'on appelait les primitifs seront considérés comme fonctionnant selon des principes plus émotionnels, ce qui justifie leur relégation dans l'espace privé ou domestique. A ce sujet, je renvoie à l'analyse que j'ai proposée au travail de l'ethnopsychologue Catherine Lutz (2004), dans la préface que j'ai consacrée à son La dépression est-elle universelle?. 
12. Dans une lettre écrite à Joseph Hookeren en 1856. Cité par Gould (1984: 40).

13. Voir à ce sujet les travaux passionnants de Bruno Latour, et plus particulièrement ses Politiques de la nature (1999).

14. Le psychologue Robert Rosenthal avait déjà démontré ce phénomène mais le considérait comme un parasite de la recherche, et donc ambitionnait de l'éradiquer; voir pour une tout autre approche Dewsbury (1992).

15. Ce que j'appelle artefact dans ce cadre est l'effet d'une visibilité de certains comportements au détriment d'autres, produit par un intérêt différencié des chercheurs.

16. Ces commentaires de Thelma Rowell sont issus d'une longue interview qu'elle m'a accordée entre le ler et le 4 juin 2003. Cette dernière a fait l'objet d'un courtmétrage, réalisé en collaboration avec Didier Demorcy, Sheep Do Have Opinions (produit par le ZKM-Zentrum für Kunst und Medientechnologie de Karlsruhe et visible dans le cadre de cette exposition).

17. Les cratéropes sont des oiseaux qui, depuis qu'ils sont observés par l'ornithologue israélien Zahavi, ont considérablement changé les opinions que nous pouvions avoir des oiseaux (cf. Zahavi et Zahavi, 1997). Voir aussi Despret (1996).

\section{Références}

Bagemihl, B. (1999) Biological Exuberance. Animal Homosexuality and Natural Diversity. Londres: Profile Books.

Darwin, C. (1981) La descendance de l'homme et la sélection sexuelle, tr. E. Barbier, d'après la seconde édition revue et augmentée par l'auteur (1874). Bruxelles: Complexe.

Despret, V. (1996) Naissance d'une théorie éthologique. La danse du cratérope écaillé. Paris: Les Empêcheurs de Penser en Rond.

De Waal, F. (1995) La politique du chimpanzé, tr. U. Ammicht. Paris: Odile Jacob.

Dewsbury, D. (1992) "Studies of Rodent-Human Interactions in Animal Psychology", in H. Davis et D. Balfour (eds) The Inevitable Bond: Examining ScientistAnimal Interaction, pp. 27-44. Cambridge: Cambridge University Press.

Fentress, J. (1992) "The Covalent Animal: on Bonds and their Boundaries in Behavioral Research", in H. Davis et D. Balfour (eds) The Inevitable Bond: Examining Scientist-Animal Interaction, pp. 44-72. Cambridge: Cambridge University Press.

Freud, S. (1912) Totem et tabou, traduction de l'allemand de S. Jankélévitch. Paris: Payot (Petite bibliothèque Payot).

Geist, V. (1971) Mountain Sheep: A Study in Behavior and Evolution. Chicago, IL: University of Chicago Press.

Gould, S.J. (1984) Quand les poules auront des dents, tr. M.F. De Palomera. Paris: Fayard.

Haraway, D. (1992) Primates' Visions. Londres: Verso.

Heinrich, B. (1991) Ravens in Winter. New York: Vintage Books.

Heinrich, B. (2000) Mind of the Raven. New York: Harper Collins.

Latour, B. (1999) Politiques de la nature, comment faire entrer les sciences sociales en démocratie. Paris: La Découverte.

Latour, B. (2001) L'espoir de Pandore, tr. D. Gille. Paris: La Découverte. 
Lefort, C. (1978) Les formes de l'histoire. Essais d'anthropologie politique. Paris: Gallimard.

Lestel, D. (1996) L'animalité. Essai sur le statut de l'humain. Paris: Hatier (collection Optiques).

Lestel, D. (2001) Les origines animales de la culture. Paris: Flammarion.

Lorenz, K. (1969) L'agression, tr. V. Fritsch. Paris: Flammarion.

Lutz, C. (2004) La dépression est-elle universelle? Paris: Les Empêcheurs de Penser en Rond.

Marx, K. (1973) “Lettre de juin 1862”, in K. Marx Lettres sur les sciences de la nature. Paris: Editions Sociales. (Orig. pub. 1862.)

Power, M. (1991) The Egalitarian: Human and Chimpanzee. Cambridge: Cambridge University Press.

Rowell, T. et Rowell, C.A. (1993) "The Organization of Feral Ovies Aries Ram Groups in the Pre-rut Period", Ethology 95: 213-32.

Russell, B. (1961) Histoire de mes idées philosophiques, tr. G. Auclair. Paris: Gallimard.

Strum, S. (1990) Voyage chez les babouins, tr. F. Simon Duneau. Paris: Eschel.

Strum, S. et Fedigan, L. (2000) Primate Encounters: Models of Science, Gender and Society. Chicago, IL: University of Chicago Press.

Todes, D. (1988) “Darwin's Malthusian Metaphor and Russian Evolutionary Thought, 1859-1917", Isis 78: 537-51.

Varikas, E. (2000) "Naturalisation de la domination et pouvoir légitime dans la théorie politique classique", in D. Gardey et I. Lowÿ (eds) L'invention du naturel, pp. 89-108. Paris: Editions des Archives contemporaines.

Zahavi, Am. et Zahavi, Av. (1997) The Handicap Principle: a Missing Piece of Darwin's Puzzle. Oxford: Oxford University Press. 